\title{
Defining blast loading 'zones of relevance' for primary blast injury research: A consensus of injury criteria for idealised explosive scenarios
}

\author{
J.W. Denny ${ }^{1}$ A.S. Dickinson ${ }^{1}$ G.S. Langdon ${ }^{2}$ \\ ${ }^{1}$ Department of Mechanical Engineering, University of Southampton, Southampton, SO17 1BJ, UK. \\ ${ }^{2}$ Department of Civil and Structural Engineering, University of Sheffield, Mappin Street, Sheffield, S1 3JD, UK.
}

Corresponding Author:

Dr Jack Denny Room 2019, Building 5, Highfield Campus, University of Southampton, SO17 1BJ

E-mail: Jack.Denny@soton.ac.uk Tel: (+44) 02380592862

\begin{abstract}
Blast injuries remain a serious threat to defence and civilian populations around the world. 'Primary' blast injuries (PBIs) are caused by direct blast wave interaction with the human body, particularly affecting air-containing organs. Despite development of blast injury criteria since the 1960 s, work to define blast loading conditions for safety limits, protective design and injury research has received relatively little attention. With a continued experimental focus on PBI mechanisms and idealised blast assumptions, meaningful test outcomes rely on appropriate simulated conditions. This paper critically evaluates existing predictive criteria for PBIs (grouped into those affecting the auditory system, pulmonary injuries and brain trauma) as a function of incident blast wave parameters, assuming idealised air blast scenarios. Analysis of the multi-injury criteria reveals new insights and understanding. It showed that blast conditions of relevance to realistic explosive threats are limited and they should be an important consideration in the design of clinical trials simulating blast injury. Zones of relevance for PBI research are proposed to guide experimental designs and compare future data. This work will prove valuable to blast protection engineers and clinical researchers seeking to determine blast loading conditions for safety limits, protective design requirements and injury investigations.
\end{abstract}

\section{Keywords}

Blast injury criteria, Primary blast injury, Traumatic brain injury, Shock tube, Animal models, blast injury 


\section{Introduction}

In 2019, a recorded 29,499 casualties were caused by explosive weapons globally, of which $66 \%$ were civilians, increasing to $90 \%$ in urban areas [1]. In recent conflicts, blasts account for approximately $80 \%$ of modern combat injury [2]. Blast injuries caused by conflict and terrorism remain a global challenge, posing a serious and ongoing threat to defence and civilian populations around the world. As a result, there is a continuing need for blast injury research to develop understanding, therapeutics and protection.

Blast injuries are a complex type of physical trauma resulting from direct or indirect exposure to an explosion. Explosions can cause human injury through a number of mechanisms (shock wave transmission, penetrating fragments and blunt impacts etc.) which can exert a variety of mechanical stresses on different tissues.

Traditionally, these effects have been classified from primary to quinary blast injury mechanisms, with the initial descriptions credited to Zuckerman during the Second World War [3]; a more detailed description was subsequently produced by the US Department of Defense in 2008 [4]. Although people often suffer from a combination of blast injury mechanisms, or 'polytrauma', researchers typically investigate injury mechanisms in isolation. For example, it is estimated that $58 \%$ of all experimental blast injury research between 2000-2019 investigated primary blast injury (PBI) mechanisms [5].

This review focusses specifically on injuries caused by exposure to blast overpressure, known as 'primary' blast injuries (PBIs). By their definition, the likelihood, severity and extent of PBls are intrinsically related to the blast wave exposure parameters, namely, the peak overpressure, $\mathrm{Pi}_{\mathrm{i}}(\mathrm{kPa})$ and positive phase duration, $\mathrm{t}^{+}(\mathrm{ms})$, which depend on the explosive scenario. Air-containing organs such as the lungs, gastro-intestinal tract and ears are particularly vulnerable to $\mathrm{PBI}$ due blast wave interaction with the body inducing rapid, pressure gradients in tissues $[6,7]$. PBIs can be serious, for example, $47 \%$ of fatalities in the terrorist bombings in Northern Ireland were attributed to pulmonary barotrauma (blast lung) [8]. More recently, blast-related brain injuries have become an increasing problem for military personnel [2] where it is estimated that nearly $60 \%$ of military patients exposed to blast in recent conflicts were diagnosed with some form of traumatic brain injury [9]. Blast traumatic brain injury (bTBI) has been linked to blast overpressure exposure $[10,11]$ although the underlying mechanisms are not well understood, making it a focus of recent research activity. Between 2000-2019, it is estimated that almost half of all public investment into blast injury-related research focussed on bTBI [5].

Blast injury research aims to generate new understanding to predict, prevent or mitigate blast injuries and to support the development of therapeutics and protection to improve health outcomes. Contemporary studies have concentrated on replicating injuries in the laboratory using in vitro and in vivo models, and it is estimated that between $2000-2019$, over $65 \%$ of public research investment into blast injury science was awarded to pre-clinical, experimental studies [5]. With bTBI remaining a research priority, future experimental work will continue to examine $\mathrm{PBI}$ mechanisms. 
Experimental PBI studies typically expose test subjects to blast waves from explosive testing or simulated conditions using laboratory equipment. The rationale behind loading conditions varies and is inevitably influenced by facility and equipment capabilities. Despite a wide range of possible loading conditions, blast loading conditions adopted in injury research should be clinically relevant and appropriate for the injury type being investigated, that is, the loading is sufficient but not excessive - to initiate the injury of interest and also relate to real-world explosive threats.

A number of PBI criteria have been proposed and developed to predict injury outcome as a function of key blast wave parameters. Injury criteria help us to understand the relationship between blast exposure and pathophysiological response and can be used to inform the design of clinically relevant loading conditions within research, plus define appropriate safety distances and protection measures.

For a given explosive scenario, a range of PBls are possible depending on a person's blast exposure. At present, there is limited guidance for designing appropriate loading conditions for PBI experimental work and there have been few reviews concerning $\mathrm{PBI}$ criteria or exposure thresholds in recent years. PBI criteria are disjointed and apply to specific injury types or anatomical regions. Similarly, previous reviews surrounding PBI criteria have focussed on injury types in isolation e.g. pulmonary (lung) injury [12] or the brain [13]. This makes it challenging to understand the range of blast loading conditions responsible for the full spectrum of possible PBls (e.g. mild hearing loss through to severe lung injury or risk of fatality), and spatially, where such conditions would occur for a given explosive scenario. Without broader comparison between injury criteria, it is difficult to establish ranges of loading conditions that are appropriate for testing.

With a continued socioeconomic and humanitarian need for research focus on PBI mechanisms, it remains important that simulated blast loading is designed appropriately to ensure meaningful outcomes. This paper reports a critical review of the literature, considering existing methods and predictive criteria for PBIs as a function of incident blast wave parameters, assuming idealised air blast scenarios. Through unique combination and analysis of multiple PBI criteria, the review's objective was to identify and define 'zones' of relevant blast wave parameters that are clinically appropriate for the investigation of different PBIs. Combined criteria are also compared to blast wave parameters corresponding to realistic explosive threats to provide context and further define relevant loading conditions. Finally, the interpretation of mapping zones of relevant blast wave parameters is discussed within the context of blast injury research and recommendations are proposed for developing future injury criteria.

\section{Blast Wave Simulation in Blast Injury Research}

Following an explosive detonation, a violent expansion of gases force surrounding air outwards at supersonic speeds, forming a layer of propagating, compressed air known as a blast wave. In an ideal scenario, blast wave propagation in unobstructed, 
free-air theoretically follows a waveform pattern known as the Friedlander function [14] (eq. 1):

$$
P_{i}(t)=P_{i}\left[1-\frac{t-t_{a}}{t^{+}}\right] \exp \left\lfloor\frac{-A\left(t-t_{a}\right)}{t^{+}}\right\rfloor
$$

where $\mathrm{P}_{\mathrm{i}}(\mathrm{t})$ is the incident overpressure at time $\mathrm{t}(\mathrm{kPa}) ; \mathrm{P}_{\mathrm{i}}$ is the peak incident pressure $(\mathrm{kPa}) ; \mathrm{t}^{+}$is the positive phase duration (ms); $\mathrm{A}$ is the decay coefficient (dimensionless); and ta is the arrival time (ms). An idealised blast wave is characterised by an instantaneous increase in pressure to the 'peak overpressure' $\left(\mathrm{P}_{\mathrm{i}}\right)$ (above atmospheric), which decreases over a time known as the 'positive phase duration' $\left(\mathrm{t}^{+}\right)$. This is then followed by a period of underpressure before normalisation occurs (Fig. 1).

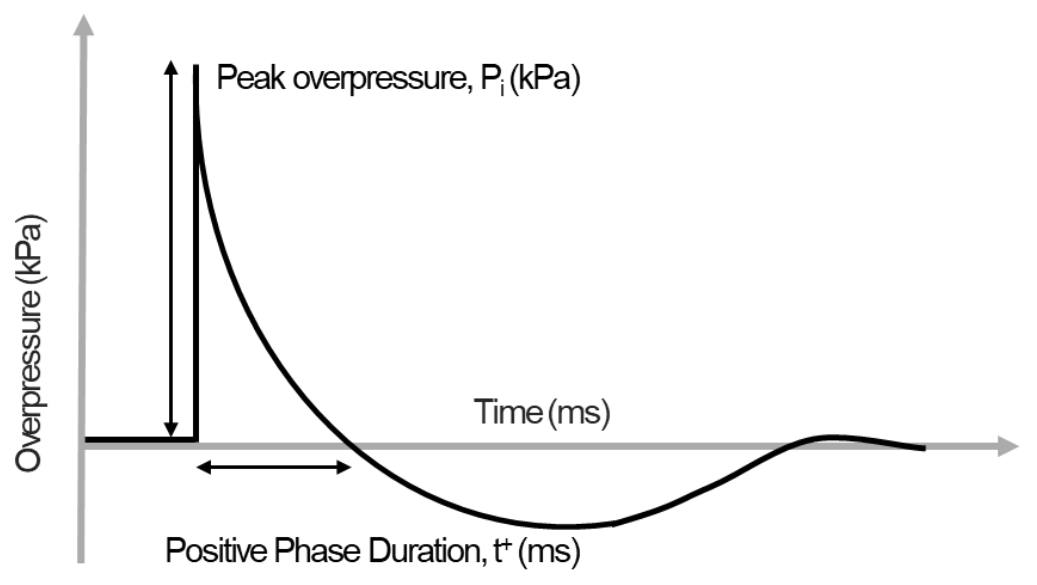

Fig. 1: A pressure-time history of an ideal Friedlander type blast wave.

Importantly, the likelihood, severity and extent of PBIs are intrinsically related to the blast wave parameters a person is exposed to, namely, the peak overpressure, $P_{i}$ $(\mathrm{kPa})$ and positive phase duration, $\mathrm{t}^{+}(\mathrm{ms})$. These key blast wave parameters depend on the explosive scenario i.e. the mass of the explosive threat, $\mathrm{W}(\mathrm{kg})$ and the distance from the detonation, or 'stand-off distance', R (Fig. 2). Furthermore, these parameters also depend on the position of the detonation with respect to the ground. When explosions are isolated from the ground or other objects, the blast wave travels away from the charge in a spherical manner (Fig 2a). When explosions occur at the ground surface, incident and reflected shock waves merge effectively instantly, forming a single hemispherical shock front with approximately twice the energy of a spherical 'free air' detonation (Fig 2b).

Extensive air-blast experimentation has been undertaken since the 1950s with alternative equations proposed to calculate blast wave quantities, including Brode [15], Kingery and Bulmash [16] and Hopkinson-Cranz [17,18]. Equations to predict incident blast wave parameters resulting from spherical air detonations and hemispherical ground detonations were developed by Kingery and Bulmash [16], who compiled explosive testing data with charge masses ranging from less than $1 \mathrm{~kg}$ to over $400,000 \mathrm{~kg}$. They developed curve-fitting techniques to represent the data with high-order polynomial equations, which have been automated in the computer program ConWep [19]. These equations are widely accepted for engineering 
predictions to determine free-field (unobstructed) overpressures and loads on structures.

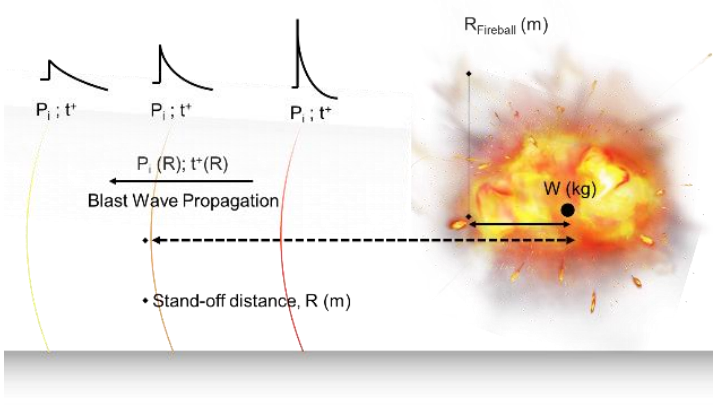

(a) Above ground, air detonation

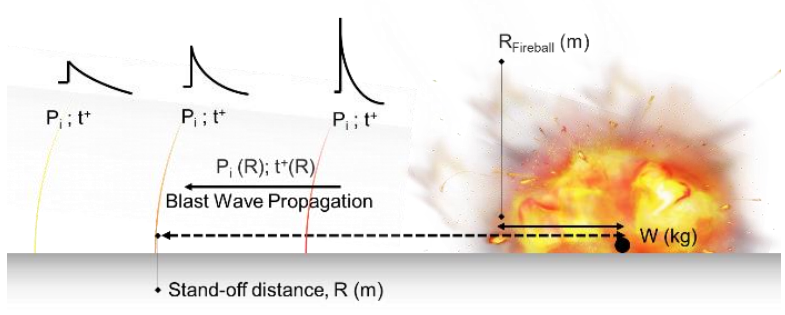

(b) Ground surface detonation

Fig. 2: Air blast wave parameters depend on where the detonation occurs with respect to the ground surface.

These predictive methods calculate idealised blast wave parameters, which can be expected when a detonation occurs in an open field in the absence of any obstructions or reflecting surfaces (Fig. 2). In reality, explosions in urban and transport environments lead to complex waveforms due to additional reflections and confinement, which can greatly modify, and typically amplify blast overpressures. Although there are many other circumstances and factors that can influence blast effects, the importance and relationship between blast wave parameters and resulting PBIs remains irrefutable. Furthermore, despite the limitations of idealised blast wave models, experimental approaches typically simulate these conditions.

The loading effects from an explosion can be simulated in numerous ways. For blast injury science, laboratory equipment can be used to simulate the varying injury mechanisms expected from an explosive detonation. A comprehensive overview and examples of different loading methodologies and equipment that can be used for blast injury research is provided by Nguyen et al. [20], which includes shock wave generation, high velocity impact and accelerations.

Experimental research on PBIs requires the generation of blast waves, which traditionally involves full-scale arena explosive testing. Full-scale explosive testing is expensive and requires specialist facilities so researchers often simulate blast using technological solutions such as air- or explosively-driven shock tubes, or numerical modelling. Some of these methods, if appropriate, can provide control of the physical components of blast by simulating idealised Friedlander-type blast waves analogous to open field explosive tests. A comprehensive overview of these approaches are described by Bass et al. [13].

Blast injury research requires an interdisciplinary approach although typically remains a clinically-driven field with limited blast engineering input, which has led to numerous systems claiming to generate 'blast', 'shock wave' and blast injury; unfortunately, many of them reproduce unrealistic conditions and/or clinically irrelevant injuries. For example several experimental devices have been described recently that use ultrasound (i.e. shock wave lithotripsy) $[21,22]$ or other means of generating overpressure, such as microwave [23] or laser [24].The resulting shockwaves do not have the physical properties of a blast (i.e. an explosion- 
generated shockwave) and do not replicate features of blast injuries observed in individuals exposed to blast [25]. Blast conditions generated by compressed air shock tubes, or blast tubes that use explosive charges, not only vary widely, but they are also limited in their capacity to reproduce conditions that represent real-world explosive environments. It is therefore important that the limitations of facilities and equipment are acknowledged and that loading conditions are placed within the context of real-world explosions.

Ideally, simulated blast loading regimes should be designed to be clinically relevant and appropriate for the injury type being investigated, i.e. powerful enough to initiate the injury without causing higher severity injury or fatality. As experimental methods continue to simulate idealised blast waves, the design of loading conditions can be informed by reference to existing predictive PBI criteria.

\section{Primary Blast Injury (PBI) Criteria}

A large goal of blast injury research has been the development of criteria to predict injury on a probabilistic basis. Blast injury criteria are helpful for the development of protective equipment, providing guidelines for risk and informing decisions in occupational health policies on acceptable exposure. Within research, injury criteria for $\mathrm{PBI}$ can also help us to understand the relationship between blast exposure and pathophysiological response.

A number of injury criteria have been proposed over the years to predict or relate blast injury outcomes to the blast conditions a person is exposed to. The most widely reported $\mathrm{PBI}$ criteria are applicable to idealised blast waves, which develop in openfield explosion scenarios. Alternative injury models have been developed that are also applicable to non-ideal and complex blast waveforms, such as the Axelsson BTD model [26] and the Weathervane SP model [27], reviewed in greater detail by Teland [12]. These alternative models allow the input of non-ideal blast waves, such as those that develop in urban settings, and predict the degree of injury in terms of a combined 'Adjusted Severity of Injury Index' (ASII). In comparison to injury criteria for idealised blast wave inputs, these models have increased complexity and their accuracy is unknown [12]. Furthermore, despite the limitations of idealised loading inputs for injury criteria, most research facilities and equipment continue to generate or assume such conditions.

Gas-containing organs such as those in the auditory system, the respiratory system (lungs) and gastrointestinal (GI) tract are more susceptible to PBI than solid organs like the brain, so were initially the focus of research $[28,29]$. The ear is recognised as one of the major organs frequently damaged from blast overpressure because the auditory system has the lowest threshold for injury when compared to the other air containing organs such as the lungs and bowel [30-32]. In the middle ear, tympanic membrane perforation can result in hearing loss, earache, vertigo, and bleeding from the external canal. Cadaver and in vivo studies have investigated tympanic membrane injuries leading to the development of injury criteria in the form of peak effective pressure thresholds (Table 1). It is also reported that temporary hearing loss can occur at pressures below the threshold for eardrum rupture [33]. These pressure thresholds relate to the maximum effective pressure, which is the highest of 
either the incident overpressure, the incident overpressure plus the dynamic pressure (stagnation pressure), or the reflected pressure. The maximum of these pressures depends on several factors including the orientation of the individual relative to the blast, the proximity of reflecting surfaces and the occurrence of jetting effects which cause pressure amplification.

Table 1: Pressure thresholds for PBI and risk of fatality have been proposed in literature.

\begin{tabular}{|l|l|}
\hline Injury Effects on Unprotected Person & Peak Effective Pressure (kPa) \\
\hline Tympanic Membrane/Ear Drum Rupture & \\
\hline Threshold for eardrum rupture & $35[33-36]$ \\
\hline $50 \%$ chance of eardrum rupture & $103[33,35]$ \\
\hline $100 \%$ of eardrums will rupture & $202[37,38]$ \\
\hline Pulmonary (Lung) Injury & \\
\hline Threshold for lung damage & $207-276[33,35]$ \\
\hline $50 \%$ fatality rate from pulmonary blast injury & $290-390[38]$ \\
\hline $95-100 \%$ fatality rate from pulmonary blast injury & $400-550[38]$ \\
\hline $50 \%$ chance of severe lung damage & $552[33,35]$ \\
\hline Fatality & \\
\hline Threshold for death & $689[33,35]$ \\
\hline $50 \%$ chance of death & $896-1,241[33,35]$ \\
\hline Near $100 \% /$ death usual & $1,379-1,723[33,35]$ \\
\hline
\end{tabular}

Early shock tube and explosive tests have indicated that human blast tolerance varies with both the magnitude and duration of the blast wave overpressure. Despite these observations, pressure thresholds (independent of duration) have also been proposed for pulmonary (lung) injury and probability of fatality (Table 1). Reported pressure ranges and thresholds show some conflicting and wide-ranging pressure thresholds, which can make it difficult to confidently design suitable loading conditions in experimental work. Reported pressure thresholds for fatality risk also conflict with the early work of Bowen et al. [29] where lethality was shown to be dependent on both duration and pressure. Inspection of pressure thresholds in Table 1 shows a general pattern whereby ear injuries are expected to occur at lower blast overpressures than pulmonary (lung) injuries, which increase with severity and likelihood of fatality with increasing overpressure.

\section{$P B I$ criteria for pulmonary injury}

The most widely used and recognised injury criteria for blast exposure are those developed by Bowen et al. [29] for pulmonary (lung) injury risk. The model is based on 2097 tests with 13 animal species positioned mostly in front of a reflecting surface using both a shock tube and high explosive charges. Interspecies scaling was employed to account for differences in the mass of the various animal species to convert all blast doses to a human-equivalent level. The probability of human survivability, fatality and injury threshold when exposed to Friedlander type blast waves were developed, dependent upon peak overpressure and duration and are commonly referred to as the "Bowen curves" [29].

Fig. 3 shows the Bowen curves for a 70-kilogram man subjected to Friedlander type blast waves while stood near a reflecting surface. For short positive phase durations $(<10 \mathrm{~ms})$, risk is highly dependent upon both blast positive phase duration and peak overpressure, increasing as overpressure duration or peak pressure increase. At longer durations (>30ms), risk becomes dependent upon peak pressure only. In addition to the lungs, work by Stuhmiller [45] showed that all major air containing 
organs (upper respiratory tract, lungs and gastrointestinal (GI) tract) have similar injury thresholds, suggesting that the Bowen curves have wider applicability to aircontaining organs. Despite being over 50 years old, the Bowen curves are still widely used as the standard for blast pulmonary injury predictions.

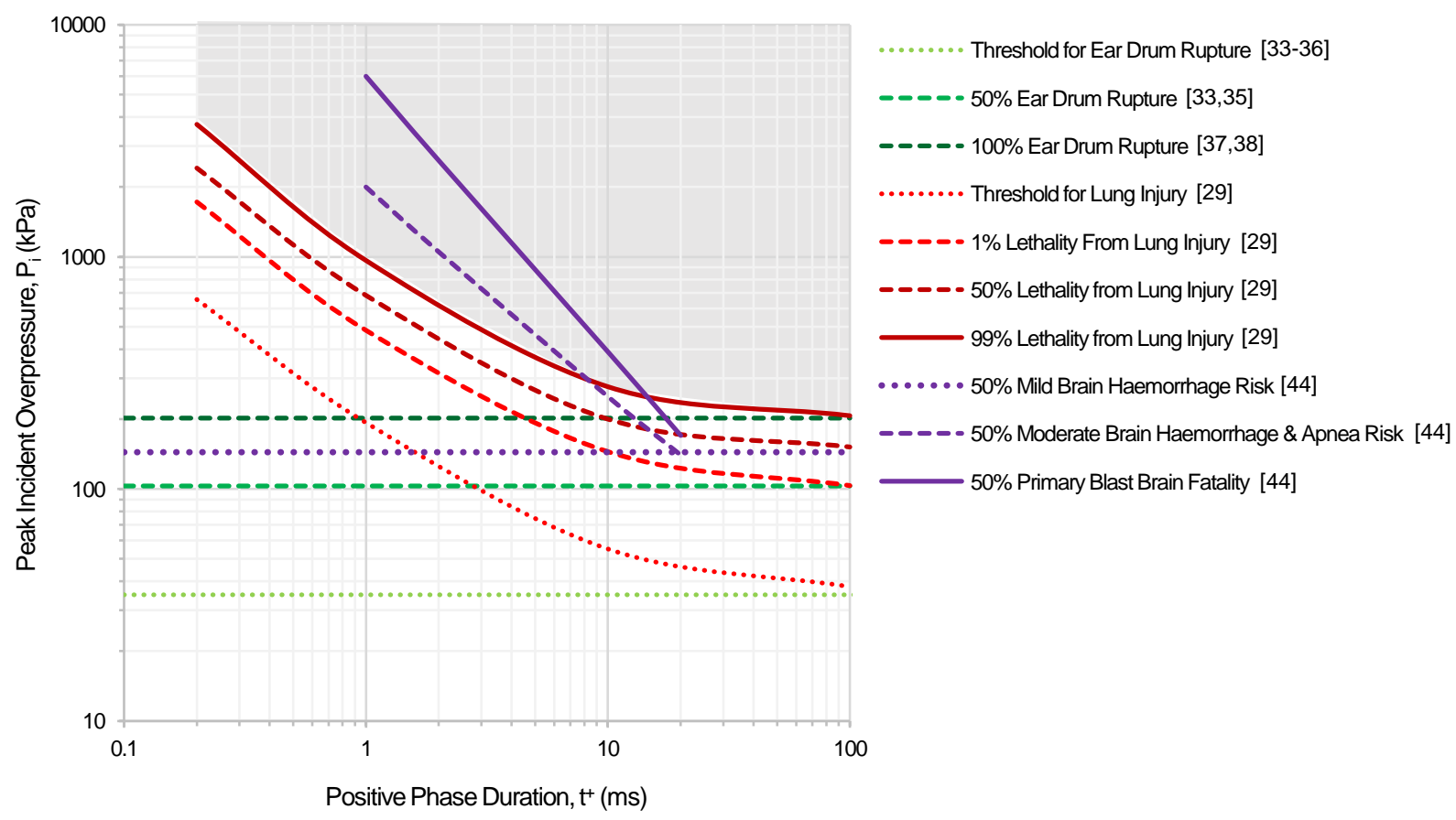

Fig. 3: Combined PBI criteria (see Table 2) to define zones of relevant blast loading conditions.

The original Bowen curves are strictly applicable to situations where a person is stood against a wall. The so-called 'pressure dose' concept was developed to extend the applicability of Bowen's model to a person in a prone position (long axis of the body parallel to the blast wave direction) and a person standing in an open field. This was possible by assuming that equivalent trauma will result if the peak reflected pressure and duration when against a reflecting surface are equivalent to the incident (side-on) pressure and duration for a body with the long axis parallel to the direction of the blast. For a body perpendicular to the blast in the open-field, the incident pressure plus the dynamic pressure (i.e. the stagnation pressure) is equated to the peak reflected pressure for equivalent trauma.

More recently, the Bowen curves have been scrutinised with some researchers proposing alternative or modified curves. Bass et al. $[39,40]$ gathered more data in order to update and improve the Bowen curves. In total, data from more than 2550 large animal experiments (including the 351 large animals from Bowen) were used in the new calculations $[39,40]$. The new curves were published in two separate articles dealing with two different regimes: short duration (<30 ms) [39] and long duration (>10 ms) [40] blast waves. Like Bowen, the Bass group found that injury outcome is highly dependent upon peak overpressure and positive phase duration in the shortduration regime, whereas limited effect of duration is observed for longer duration blasts. The updated injury curves developed by Bass et al. [39,40] for a person located near a wall are almost identical to the original curves developed by Bowen for the same scenario. 
The Bass curves were also extended to the open field and prone situation, but in a different way than the Bowen curves. For a prone situation, the extension was similar with Bass assuming (as Bowen) that the pressure dose was the incident pressure $\mathrm{p}_{\mathrm{i}}$, although there is still no data available for testing this hypothesis. For an open field situation, Bass and Bowen diverged considerably in their approach. Instead of using the incident pressure plus dynamic pressure (stagnation pressure), Bass used the reflected pressure prfrom an imaginary wall behind the subject as the pressure dose. Bass et al. [39] claimed that in the short duration regime the body itself acts as a reflecting surface. They concluded that "the pressure dose for both bodies against a reflecting surface and bodies parallel to the blast for short durations is assumed to be the reflected pressure." The Bass formula therefore considers the open field situation to be much more dangerous than Bowen (in fact, just as dangerous as being near a wall). Consequently, according to Bass, lethality in pulmonary blast injury is exactly the same for standing in an open field and standing near a wall.

Further work on pulmonary injury risk in large animals was conducted by Panzer et al. [41] and combined short and long overpressure duration data. A piece-wise linear model was used to describe injury risk dependent upon peak pressure and overpressure duration based on existing injury data from literature and did not further consider differences in orientation. More recently, van der Voort et al. [42] proposed a 'new standard' of lung injury curves based on a theoretical study of Friedlander blast waves and numerical blast simulations which remain effectively identical to the original Bowen curves for the reflected wall scenario, but deviate from the original Bowen model for the open-field scenario [42].

Overall, updates to the original Bowen curves have made relatively little difference to predictions of pulmonary injury lethality, although differing assumptions for extending the curves to different situations or orientations (e.g. prone or open field) have. Considering the recent developments of pulmonary injury curves by different researchers, there still lacks consensus or verification of injury curves applicable to the open field scenario. In general, recent modifications to pulmonary injury criteria have had the effect of increasing the risk of injury associated with the free-field scenario compared to those first proposed by Bowen.

\section{$P B I$ criteria for brain injury}

PBI criteria were initially developed for the lungs because they were more susceptible to injury, with a higher risk for fatality if the thorax is unprotected [39]. $\mathrm{PBI}$ tolerance of the brain was not investigated until relatively recently following an increase in survivable blast injuries through improved protective equipment and an increasing incidence of blast TBls. There is far less experimental data for blast neurotrauma compared with pulmonary experimentation, making development of injury criteria more challenging. However, there have been studies investigating single or a small group of different species over a large enough exposure range to develop injury risk models.

Rafaels et al. [43] developed risk curves for fatality from primary blast brain injury. It was found that the brain's $50 \%$ risk of fatality from blast occurred at overpressure values well above the $99 \%$ risk of fatality from pulmonary injuries (Fig. 3, red lines). 
This suggested that the blast level needed to cause fatality from blast exposure to the head was greater than the peak overpressure needed to cause fatality from pulmonary injury.

Further tests were undertaken by Rafaels et al. [44] to evaluate mild to severe brain injury. New data combined with previously published data were used to develop risk curves for blast neurotrauma, specifically, apnea, bleeding (of different severities) and fatality for positive-phase durations of $1 \mathrm{~ms}$ to $20 \mathrm{~ms}$. For most outcomes, tolerance to blast peak overpressure decreased with increasing positive phase duration. Apnea, bleeding, and fatality risk from blast exposure to the head were determined for peak overpressure and positive-phase duration (Fig. 3, purple lines). The $50 \%$ risk of apnea and moderate haemorrhage were similar, whereas the $50 \%$ risk of mild haemorrhage was independent of positive phase duration and required lower overpressures (144 kPa). Importantly, the blast level for $50 \%$ risk of mild brain bleeding (intracranial haemorrhage) was found to occur at overpressure values below the threshold for pulmonary injury (Fig. 3, red lines) [44].

\subsection{Analysis of Combined PBI Criteria}

A selection of PBI criteria (Table 2) are combined for analysis and plotted in Fig. 3 to examine zones of clinically relevant blast loading conditions for different PBIs. The selected PBI criteria were grouped into the following categories: (1) auditory system; (2) pulmonary (lung) injury \& lethality; and (3) brain related PBI (Table 2).

Table 2: Primary Blast Injury Criteria Combined To Define Zones Of Relevant Blast Loading Conditions for Injury Research.

\begin{tabular}{|l|l|l|}
\hline Blast Injury Area & Criteria Type & Criteria Description \\
\hline 1. Auditory System (Ears) & Pressure Thresholds & $\begin{array}{l}35 \mathrm{kPa}[33-36]-\text { Threshold for eardrum rupture } \\
103 \mathrm{kPa}[33,35]-50 \% \text { chance of eardrum rupture } \\
202 \mathrm{kPa}[37,38]-100 \% \text { of tympanic membranes will rupture }\end{array}$ \\
\hline $\begin{array}{l}\text { 2. Pulmonary (Lung) Injury \& } \\
\text { Lethality }\end{array}$ & $\begin{array}{l}\text { Pressure-Duration } \\
\text { Curves }\end{array}$ & $\begin{array}{l}\text { Bowen curves [29] - (peak overpressure-positive phase } \\
\text { duration) for primary blast pulmonary (lung) injuries assuming } \\
\text { a 70kg man stood near a wall, including: threshold (onset) and } \\
1 \%, 50 \% \text { and 99\% probabilities of fatality. }\end{array}$ \\
\hline 3. Brain-related PBI & $\begin{array}{l}\text { Pressure Threshold } \\
\text { \& Pressure-Duration } \\
\text { Functions }\end{array}$ & $\begin{array}{l}\text { Peak overpressure-positive phase duration functions for 50\% } \\
\text { probabilities of moderate brain haemorrhage \& apnea risk, and } \\
50 \% \text { risk of fatality from primary injury to the brain [44]. } \\
144 \mathrm{kPa} \text { [44] - 50\% risk of mild brain haemorrhage }\end{array}$ \\
\hline
\end{tabular}

For the auditory system, selected PBI criteria include overpressure thresholds to predict the onset and probabilities (50\% and 100\%) of tympanic membrane rupture (Table 2; Fig. 3, green lines). Predictions for the threshold (onset) and probabilities $(1 \%, 50 \%$ and $99 \%)$ of fatality from pulmonary injury utilise the Bowen et al. [29] blast overpressure-time curves (Table 2; Fig. 3, red lines). Pressure thresholds for lung damage and lethality predictions (Table 1) were not included in the analysis as a clear dependence on the blast duration has been demonstrated by researchers $[29,39,40]$. Due to lack of consensus on how to extend pulmonary injury models to the free-field scenario, the original Bowen curves [29] for a 70kg person stood near a reflective wall are selected for this analysis as this is considered to also approximately represent the open field scenario according to more recent 
researchers [39-41]. Brain-related PBI criteria developed by Rafaels et al. [44] were selected for comparison, including an overpressure threshold and overpressure-time functions to predict $50 \%$ probabilities of brain injury and fatality (Table 2; Fig. 3, purple lines).

Risk of primary ear injury is independent of blast duration, and criteria for the threshold (onset) to $100 \%$ probability of ear drum rupture permit the definition of upper and lower bounds for effective peak pressure values of interest, from 35$202 \mathrm{kPa}$. In comparison to all PBI criteria, the ear drum rupture pressure threshold $(35 \mathrm{kPa})$ represents the minimum reported pressure at which any PBIs are expected. This could therefore be perceived as a lower bound pressure for PBI research, although should be approached with caution until exposure thresholds for mild TBI are better understood.

Criteria for pulmonary injury (Bowen curves) include threshold blast loading conditions for the onset of lung injury through to $99 \%$ probability of fatality for positive phase durations $\mathrm{t}^{+}=0.2 \mathrm{~ms}-100 \mathrm{~ms}$. The threshold overpressure for lung injury decreases with positive phase duration, occurring at peak overpressures of approximately $200 \mathrm{kPa}$ at $1 \mathrm{~ms}$ duration and reducing to $55 \mathrm{kPa}$ at $10 \mathrm{~ms}$ duration (Fig. 3 ). Similarly, the overpressure giving $99 \%$ probability of fatality from lung injury decreases with blast duration, over a larger range, of approximately $965 \mathrm{kPa}$ to $275 \mathrm{kPa}$ between positive phase durations of $1-10 \mathrm{~ms}$ (Fig. 3). These criteria permit the definition of zones of blast wave parameters of interest for investigating injury to the lungs (and potentially GI tract) i.e. below the $99 \%$ risk of fatality curve and above the threshold curve.

Inspecting proposed PBI criteria for the brain, it can be seen that the $50 \%$ risk of fatality exceeds the $99 \%$ risk of pulmonary fatality for positive phase durations 1 $15 \mathrm{~ms}$ (Fig. 3), indicating that fatality from lung injury is expected before fatality from brain injuries. Similarly, criteria predicting the $50 \%$ risk of moderate brain haemorrhage or apnea exceeds the $99 \%$ risk of pulmonary fatality for positive phase durations $1-9 \mathrm{~ms}$, again indicating that fatality from lung injury is expected before moderate brain injury occurs. As these criteria exceed the $99 \%$ risk of pulmonary fatality for positive phase durations up to $9 \mathrm{~ms}$, there is arguably limited value in simulating these conditions within research as fatality from pulmonary injury would be expected for an unprotected person.

For milder brain injuries, a 50\% risk of mild intracranial bleeding occurs at overpressures of $144 \mathrm{kPa}$, independent of blast positive phase duration. In comparison to other injury criteria, this pressure threshold coincides with a $50-100 \%$ chance of ear drum rupture (Fig 3.). For positive phase durations below approximately $2 \mathrm{~ms}$, the $50 \%$ risk of mild brain bleeding occurs below the threshold for pulmonary injury (Fig. 3). Importantly, this suggests that brain injuries may occur at peak overpressure levels lower than that which causes lung injuries. For positive phase durations greater than $2 \mathrm{~ms}$, a $50 \%$ risk of mild brain bleeding coincides with blast levels capable of causing pulmonary injury (i.e. above the threshold), although remains below the $50 \%$ risk of fatality from pulmonary injury for all durations. 
Unlike PBI criteria for the ears and lungs, where clear zones of relevant blast wave parameters can be defined, for brain related injuries, minimum pressure thresholds for brain-related PBIs have not been proposed. At present, the lower bound blast exposure responsible for milder forms of brain PBIs is unknown, thus driving the research focus on understanding mild TBI and the effect of repeated, low level blast akin to military training. With a minimum exposure threshold for brain related PBIs yet to be defined, it is challenging to specify ranges of appropriate loading conditions for experimental work.

As seen in Fig. 3, different PBIs, injury severities and risk of fatality are strongly governed by the incident blast wave parameters of exposure. Comparing different $\mathrm{PBI}$ criteria, a hierarchy of injury types is observed whereby the ears are most vulnerable, followed by the lungs and brain (Fig 3.). Analysis of combined injury criteria suggests that fatality from pulmonary injury, specifically the $99 \%$ risk function proposed by Bowen, effectively acts as an upper bound of blast wave conditions of interest. While the PBI criteria analysed do not support a definitive minimum threshold, comparison between different criteria as in Fig. 3 supports zones of interest, in terms of peak overpressure and positive phase durations to guide experimental designs and compare future data. With this knowledge, simulated loading conditions can then be placed within the context of an equivalent explosive threat to ensure blast wave parameters are realistic.

\subsection{Comparing Injury Criteria to Idealised Explosive Threats}

As found in casualty and forensic reports for blast incidents [8], victims are most susceptible to PBls close to the source of detonation where blast overpressures are highest. Given an open-field explosion, the spatial extent and severities of PBIs depend on the standoff distance and explosive charge mass, which can vary widely in reality depending on the nature of the blast threat.

It is common practice in blast engineering to relate the stored energy of any explosive charge/threat to an equivalent mass of TNT based upon the ratio of the energy densities of the explosive materials. Equivalent TNT charge masses for a range of real-world explosive threats of different magnitudes, weapon type and design (e.g. manufactured and improvised) were compiled through reviewing open literature, which were grouped into blast threats and summarised in Table 3.

Table 3: Approximate TNT equivalent charge masses for realistic explosive threats.

\begin{tabular}{|l|l|l|}
\hline Blast Threat Group & Realistic Examples & $\begin{array}{l}\text { Equivalent } \\
\text { TNT Mass }\end{array}$ \\
\hline $\begin{array}{l}\text { AP Landmines \& } \\
\text { Unexploded Ordnance (UXO) }\end{array}$ & $\begin{array}{l}\text { Anti-personnel (AP) landmines- 20-200g TNT [45] } \\
\text { Mortar Round e.g. '60mm HE80'- 200g TNT [46] } \\
\text { Cluster Submunitions e.g. ShOAB-0.5 bomblets- 70g TNT [47] }\end{array}$ & 20-250g TNT \\
\hline AV Landmines \& IEDs & $\begin{array}{l}\text { Pipe Bomb - 2.3kg [48] } \\
\text { AV landmines - 3.5-7kg TNT [12] } \\
\text { Suicide belt - 4.5kg [48] } \\
\text { Suicide vest - 9kg [48] } \\
\text { Bologna railway station terrorist attack (1980) - 20kg TNT [49] } \\
\text { Rolling Luggage bomb scenario - 20-45kg [48] }\end{array}$ & 1-50kg TNT \\
\hline Vehicle-Bourne IED Threats & $\begin{array}{l}\text { Old Bailey car bomb attack, London (1973) - 80kg TNT [50] } \\
\text { VBIED/Car bomb - 227kg-454kg TNT [48] } \\
\text { Truck bomb - 4500-13000kg TNT [48] } \\
\text { Beirut International Airport truck bomb (1983)- 5455kg TNT [51] }\end{array}$ & $>75 \mathrm{~kg}$ TNT \\
\hline
\end{tabular}


In open literature, few examples are presented on the predicted spatial distribution of PBls for real world explosive threats. Holcomb et al. [52] presents a schematic illustrating the range of distances where PBIs would be expected for an open-space surface detonation of a 155-mm $100 \mathrm{~kg}$ shell. In this specific example, ranges where primary blast injuries would occur are defined, although it is unclear as to what types and severities of PBIs would be expected. Given the hierarchy of PBI criteria (Fig 3), different injury types, severity and probabilities would be expected at different standoff distances from the detonation, depending on the blast threat.

\section{Example: Comparing Idealised Air Blast Scenarios with PBI Criteria}

Incident blast wave parameters were calculated for a range of idealised explosive scenarios and plotted against injury criteria to analyse how they compare to realistic explosive threats. Empirical equations developed by Kingery and Bulmash [16], automated in the ConWep program [19], were used to calculate incident blast wave parameters (peak overpressure and positive phase duration) for each charge mass. Calculations assumed the detonation of spherical charges with masses ranging from $10 \mathrm{~g}-1000 \mathrm{~kg}$ TNT equivalent to calculate incident blast wave parameters in free space in the absence of any reflecting surfaces as a function of stand-off distance, $R$. These assumptions are acceptable when the detonation occurs above the ground surface and the incident blast wave is expected to reach the point of interest before any reflected blast waves from the ground or other structures. The range of charge masses correspond to different scale explosive threats (i.e. small unexploded remnants of war to large truck bombs) and have relevance to different blast testing capabilities at research facilities.

Calculated blast wave parameters, which map combinations of blast wave peak overpressure and positive phase durations experienced at different stand-off distances are plotted as a series of curves grouped by their respective charge mass, and are linked to predictive PBI criteria (Fig 4). Curves for the $100 \mathrm{~g}$ and $10 \mathrm{~kg}$ charge masses are plotted with corresponding stand-off distances at regular intervals. Through inspection of equivalent TNT charge masses for real-world explosive threats (Table 3 ), the $100 \mathrm{~g}$ and $100 \mathrm{~kg}$ charge sizes approximately represent antipersonnel landmine and IED size threats respectively. As explosive charge mass increases, blast waves with increased positive phase durations are generated at injury-relevant peak overpressures. Importantly, this means that larger blast threats tend to have increased likelihood of causing more serious pulmonary (lung) injuries as pressure thresholds are reduced with increasing positive phase durations. It can also be seen that with increasing charge mass (and blast wave positive phase duration), lung injury criteria converge and begin to overlap with ear and brain injury criteria.

For a given explosive charge mass, the stand-off distance at which different injury types and risk of PBIs can be analysed through comparison of PBI criteria and blast wave parameters plotted in Fig. 4. For a $100 \mathrm{~g}$ charge mass for example, there is a $50 \%$ risk of fatality from pulmonary injury at a $0.5 \mathrm{~m}$ stand-off distance, whereas at a $1 \mathrm{~m}$ stand-off, no lung injuries would be expected (below pulmonary threshold) but 
there would be a near $100 \%$ risk of ear drum rupture (Fig. 4). At a $3 \mathrm{~m}$ standoff however, effectively no PBIs would be expected given the absence of any minimum threshold criteria for brain-related injury. In contrast, for a 10kg charge mass, a $99 \%$ risk of fatality from pulmonary injury would be expected up to a stand-off distance of $3 \mathrm{~m}$, and lung injuries expected between 3-7m stand-off. Spatially, it can be seen that larger blast threats inflict PBIs over a larger distance from the detonation compared to smaller detonations. For smaller explosive threats (i.e. $100 \mathrm{~g}$ TNT), it can be seen that the range of stand-off distances where PBls are of interest is relatively limited (i.e. 0.5-2m). With limited ranges of stand-off distances that are sufficient to cause PBIs, loading conditions should be designed carefully to ensure that they are clinically relevant for the injury type of interest.

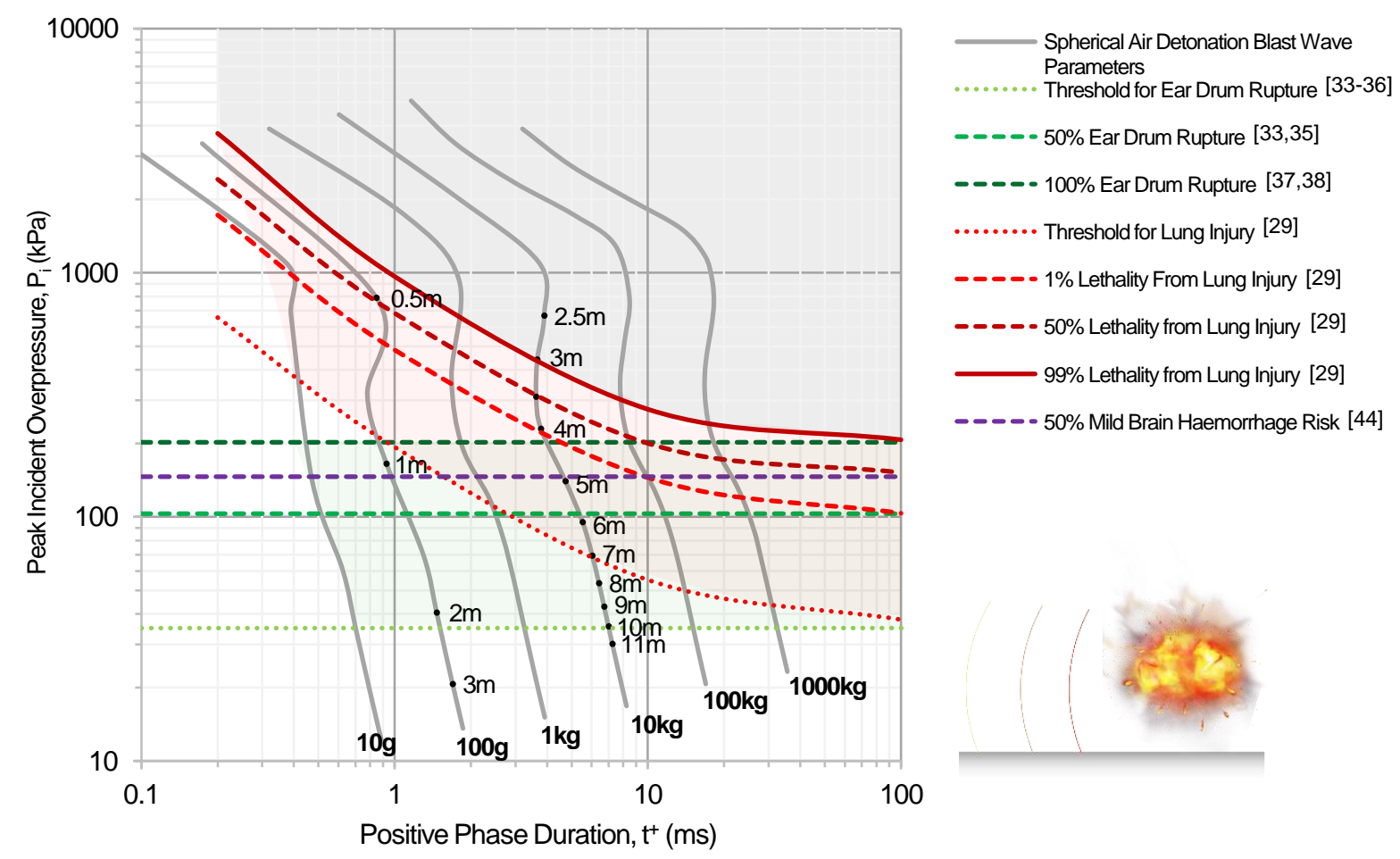

Fig. 4: Analysing PBI criteria with respect to blast wave parameters resulting from spherical air detonations at different stand-off distances.

Considering the range of blast wave parameters associated with realistic air detonations, there is a finite region of realistic positive phase durations. For example, inspection of Fig 4. suggests that there is limited relevance in simulating blast waves with positive phase durations below $0.4 \mathrm{~ms}$ as this corresponds to either very small explosive threats $(<10 \mathrm{~g}$ TNT) or larger threats at close stand-off distances that would cause fatality. Similarly, simulating positive phase durations in excess of $20 \mathrm{~ms}$, effectively models very large explosive detonations (>100kg TNT) which arguably occur less frequently.

Through plotting both PBI criteria and loading conditions that correspond to equivalent explosive scenarios, zones of blast wave parameters can be further defined that are both clinically-relevant (to the PBI of concern) and realistic (corresponding to real-world threats). Defining such zones as in Fig. 4 can be useful to inform experimental approaches (e.g. shock tube or arena testing) and guide the 
design of simulated loading conditions within injury-related research to ensure they are both realistic and relevant for the injury type. For example, understanding of expected PBIs at different stand-off distances for a known charge size is useful for researchers when designing explosive arena (open-field) tests. Comparison of PBI criteria with equivalent blast threats further highlights the importance of fully reporting both pressure and duration values within studies as this has significant implications for both the scale of the equivalent explosive threat that is being simulated and the nature and severity of PBls expected.

\section{Discussion}

The present study's analysis is limited to PBI criteria (excluding all other blast injury mechanism types) that are applicable to idealised (Friedlander waveform) blast scenarios. Within this scope, a number of limitations have been identified within the context of current approaches used in injury-related research.

\section{Blast interaction effects and factors affecting loading on humans}

Although the PBI criteria discussed in this study are based on incident (free-field) blast wave parameters, which can be readily measured using pressure sensors in experiments, a number of factors can influence the actual loading that is experienced by a human, and the potential for inflicting PBls. Actual pressure histories acting on a person depend on complex blast interaction effects due to different anatomical geometries, projected areas, body orientations and the relative angle of incidence with respect to the blast wavefront. When a blast wave interacts with the human body, reflection and stagnation occurs, generating a pressure loading profile higher than the reported incident conditions, which are effectively point measurements in free space. Furthermore, the effect of protective equipment and armour on vulnerability to PBls is not well understood with researchers suggesting that it can both amplify and decrease protection from different primary injuries [13]. Such interaction effects should be acknowledged when defining new injury criteria or replicating blast effects in injury studies. Future injury studies should aim to characterise not only the incident blast loading environment, but also pressure histories at multiple surface locations on the test subject to quantify the actual loading effects associated with injury outcomes.

\section{Blast exposure thresholds for 'mild' brain-related PBIs}

This study has highlighted that further work is needed to define thresholds corresponding to brain $\mathrm{PBI}$, in particular to define criteria and exposure thresholds for milder forms of bTBI to improve confidence in selecting clinically-relevant loading parameters. As more is understood about bTBI, it will become possible to further define thresholds for TBI-related injuries as a function of blast overpressure exposure.

\section{Interrelated, poly-injury effects}

$\mathrm{PBI}$ criteria identified in this study concern the probability of specific injury types occurring in isolation. It is currently unknown how different PBls influence, or possibly contribute to the risk of developing other PBI types. Similarly, it is not 
understood how different blast injury mechanisms (i.e. secondary fragmentation wounds or tertiary blunt impacts) contribute to the development and risk or PBIs.

\section{Understanding and capacity to model 'complex' blast wave profiles}

Although open-space explosions predominated in previous conflicts, explosive detonations are increasingly occurring in urban settings due to the nature of terrorist attacks in densely populated regions and the shifting nature of conflict into more urban areas [1]. This presents complex, and highly-variable geometries and layouts that can significantly modify blast loading conditions and the implications for PBIs. Explosive effects are substantially different in closed spaces, where blast waves deflect, ricochet, and coalesce [52]. Enclosure magnifies their destructive power and also generates large numbers of secondary fragments through the breakup of structures and vehicles. Even in relatively simple open-space scenarios, detonations above the ground also create subsequent blast waves due to ground reflection, which could potentially cause injury.

At present, PBI criteria are limited to estimating the likelihood or exposure threshold for a person subjected to a single, highly idealised blast pressure profile from detonations occurring in a free-field environment. Attempts to apply these PBI criteria rely on the use of peak pressure and duration, which may not be appropriate for complex waveforms and have not been validated at this time. This presents several challenges for applying existing PBI criteria and defining requirements for new injury criteria. In the case of modifying existing PBI criteria, future work could explore the extent to which scaling methods could be developed to translate criteria from idealised to 'complex' blast wave scenarios. Alternatively, it may be necessary to investigate and define entirely new PBI criteria for complex blast waveforms. For either strategy, the infinite range of possible blast environments (e.g. different urban layouts) will make any developments very challenging. Furthermore, any advancements in knowledge of these complex loading cases will require careful restriction to avoid informing terrorist activity.

Due to these complexities and inherent variability of pressure profiles arising in realistic blast scenarios, some could argue that there is limited benefit in further refining or developing existing injury criteria that are based on idealised blast wave assumptions. However, given the nature and capabilities of experimental facilities (e.g. shock tubes and blast arena trials), future research will continue to assume or model idealised Friedlander type blast waves. As a result, the need to better understand and specify clinically relevant idealised loading regimes will remain.

\section{Reviewing loading conditions in previous $P B I$ research}

Despite the inherent limitations of injury criteria assuming idealised blast waves, analysis of combined $\mathrm{PBI}$ criteria with blast wave parameters corresponding to different blast threats allowed zones of relevant loading conditions to be defined, which are useful for informing experimental design and support selection of sensible loading conditions.

This approach could be used to review prior research to evaluate the clinical relevance of loading conditions simulated in prior studies and place them within the context of equivalent explosive threats. This would also provide a systematic method 
of comparing multiple injury studies and assessing whether findings and methods were observed under similar loading conditions, facilitating meta-analysis. The ability to compare findings from prior studies performed under similar loading conditions would promote financial savings through reduced duplication.

Appropriate simulation of explosive loading is important to ensure that blast injury and protection research generates meaningful findings that can translate into improved protection, clinical treatments, health outcomes and quality of life for defence and civilian populations. In the long term, better designed experimental work adopting blast wave loading with increased clinical relevance will lead to higher impact outcomes and cost-effective development of clinical advancements.

\section{Conclusion}

This paper presents a critical compilation of existing PBI criteria to examine the extent of blast wave loading conditions that are clinically-relevant for investigating a range of PBls. Analysis of broader injury criteria, and comparison to idealised blast wave parameters associated with a range of explosive threats enables new oversight and understanding of relevant 'zones' of blast loading parameters for injury-related research. Analysis showed that blast conditions of relevance to realistic explosive threats are limited and they should be an important consideration in the design of clinical trials simulating blast injury. Zones of relevance for primary blast injury research work are proposed to guide experimental designs and compare future data. This work will prove valuable to blast protection engineers and clinical researchers seeking to determine blast loading conditions for safety limits, protective design requirements and perform clinical injury investigations. This review has highlighted knowledge gaps surrounding PBI criteria and demonstrated the need for comprehensive guidance for experimentally simulating blast waves within blast injury research. Finally, knowledge gaps and recommendations for the future development of $\mathrm{PBI}$ criteria were discussed in the context of complex blast waveforms arising in non-ideal explosive environments.

\section{Conflicts of Interest}

None

\section{Funding}

EPSRC Doctoral Prize

The Royal Academy of Engineering (RAEng) (award no. RF/130)

\section{Ethical Approval}

Not required 


\section{References}

[1] Action on Armed Violence (AOAV). Explosive violence in 2019. AOAV 2020. https://aoav.org.uk/2020/explosive-violence-in-2019/.

[2] Owens, Brett D.; Kragh, John F. Jr; Wenke, Joseph C.; Macaitis, Joseph; Wade, Charles E.; Holcomb JB. Combat Wounds in Operation Iraqi Freedom and Operation Enduring Freedom. J Trauma 2008;64:295-9. https://doi.org/10.1097/TA.0b013e318163b875.

[3] Zuckerman S. Discussion on the problem of blast injuries. Proc R Soc Med $1941 ; X X X I V: 171-92$.

[4] US Department of Defense (DoD). DoD Directive 6025.21E: Medical Research for Prevention, Mitigation, and Treatment of Blast Injuries. 2006.

[5] Denny JW, Brown RJ, Batchelor J. Sizing Up Blast Injury Research: A TransDisciplinary Evidence-Base To Maximise Impact \& Relevance. 4th Int. Forum Blast Inj. Countermeas. 2019, Washington, D.C.: 2019.

[6] Mellor SG, Cooper GJ. Analysis of 828 servicemen killed or injured by explosion in Northern Ireland 1970-84: the Hostile Action Casualty System. Br J Surg 1989.

[7] Bull AMJ, Clasper J, Mahoney PF. Blast Injury Science and Engineering: A Guide for Clinicians and Researchers. Springer, 2016; 2016.

[8] Frykberg ER, Tepas JJ. Terrorist Bombings: Lessons Learned From Belfast to Beirut. Ann Surg 1988.

[9] Okie S. Traumatic brain injury in the war zone. N Engl J Med 2005;352:20437. https://doi.org/10.1056/NEJM200508113530621.

[10] Martin EM, Lu WC, Helmick K, French L, Warden DL. Traumatic brain injuries sustained in the afghanistan and Iraq wars. Am J Nurs 2008;108:40-7. https://doi.org/10.1097/01.NAJ.0000315260.92070.3f.

[11] The Management of Concussion/mTBI Working Group. VA / DoD CLINICAL PRACTICE GUIDELINE FOR MANAGEMENT OF CONCUSSION / MILD TRAUMATIC BRAIN INJURY The Management of Concussion / mTBI Working Group. 2009. https://doi.org/10.1682/JRRD.2009.06.0076.

[12] Teland JA. Review of blast injury prediction models. FFI-rappor. Norwegian Defence Research Establishment (FFI); 2012.

[13] Bass CR, Panzer MB, Rafaels KA, Wood G, Shridharani J, Capehart B. Brain injuries from blast. Ann Biomed Eng 2012;40:185-202. https://doi.org/10.1007/s10439-011-0424-0.

[14] Friedlander FG. The Diffraction of Sound Pulses. II. Diffraction by An Infinite Wedge. Proc R Soc London A Math Phys Eng Sci 1946;186:344-51.

[15] Brode HL. Numerical Solution of Spherical Blast Waves, RM-1363-AEC. Santa Monica, California: 1954.

[16] Kingery CN, Bulmash G. Airblast Parameters From TNT Speherical Air Burst and Hemispherical Surface Burst, Technical Report ARBRL-TR-02555. 1984.

[17] Hopkinson B. British Ordnance Board Minutes 1915. 
[18] Cranz C. Lehrbuch der Ballistik. Berlin: Springer-Verlag; 1926.

[19] Hyde DW. ConWep: Conventional Weapons Effects (Application of TM 5-8551) 1992.

[20] Nguyen TT, Pearce AP, Carpanen D, Sory D, Grigoriadis G, Newell N, et al. Experimental platforms to study blast injury. J R Army Med Corps 2019;165:33-7. https://doi.org/10.1136/jramc-2018-000966.

[21] Divani, Afshin A., Murphy, Amanda J., Meints, Joyce, Sadeghi-Bazargani, Homayoun, Nordberg, Jessica, Monga, Manoj, Low, Walter C., Bhatia, Prerana M., Beilman, Greg J., SantaCruz KS. A Novel Preclinical Model of Moderate Primary Blast-Induced Traumatic Brain Injury. J Neurotrauma 2015;32:1109-16.

[22] McCabe JT, Moratz C, Liu Y, Burton E, Morgan A, Budinich C, et al. Application of high-intensity focused ultrasound to the study of mild traumatic brain injury. Ultrasound Med Biol 2014;40:965-78. https://doi.org/10.1016/j.ultrasmedbio.2013.11.023.

[23] IGARASHI Y, MATSUDA Y, FUSE A, ISHIWATA T, NAITO Z, YOKOTA H. Pathophysiology of microwave-induced traumatic brain injury. Biomed Reports 2015;3:468-72. https://doi.org/10.3892/br.2015.454.

[24] Nakagawa A, Keisuke O, Kiyonobu K, Goda D, Arafune T, Toshikatsu W. Mechanism of Traumatic Brain Injury at Distant Locations After Exposure to Blast Waves: Preliminary Results from Animal and Phantom Experiments. Intracranial Press. Brain Monit. XV, vol. 122. Part of th, 2016. https://doi.org/10.1007/978-3-319-22533-3.

[25] Cernak I. Understanding blast-induced neurotrauma: how far have we come? $2017 ; 2$.

[26] Axelsson H, Yelverton JT. Chest Wall Velocity as a Predictor of Nonauditory Blast Injury in a Complex Wave Environment. J Trauma 1996;40:31S-37S.

[27] Li E, Yoshinaka A, Josey T. Weathervane: a single point model for blast injury approximations. 20th Symp. Mil. Asp. Blast Shock, Oslo: 2008.

[28] Clemedson CJ. Blast Injury. Physiol Rev 1956;36:336-54.

[29] Bowen IG, Fletcher ER, Richmond DR. Estimate of Man's Tolerance to the Direct Effects of Air Blast. Washington, D.C.: 1968.

[30] Garner J BSJ. Mechanisms of injury by explosive devices. Anesth Clin 2007;25:147 - 160 .

[31] Harrison CD, Bebarta VS, Grant GA. Tympanic membrane perforation after combat blast exposure in iraq: A poor biomarker of primary blast injury. J Trauma - Inj Infect Crit Care 2009;67:210-1. https://doi.org/10.1097/TA.0b013e3181a5f1db.

[32] Mrena R, Pääkkönen R, Bäck L, Pirvola U, Ylikoski J. Otologic consequences of blast exposure: A finnish case study of a shopping mail bomb explosion. Acta Otolaryngol 2004;124:946-52. https://doi.org/10.1080/00016480310017045.

[33] US Department of Defense (DoD). UFC 3-340-02, "Structures To Resist The Effects Of Accidental Explosions." Washington, D.C.: 2008. 
[34] Hirsch FG. Effects of overpressure on the ear: a review. Ann N Y Acad Sci 1968;152:147-62.

[35] Owen-Smith MS. Hunterian lecture 1980: A computerised data retrieval system for the wounds for war: The Northern Ireland casualties. Ournal R Army Med Corps 1981;127:31-54.

[36] Beveridge A. Forensic Investigation of Explosions. Boca Raton: CRC Press; 1998. https://doi.org/https://doi.org/10.1201/b11938.

[37] Jensen $\mathrm{JH}$, Bonding P. Experimental pressure induced rupture of the tympanic membrane in man. Acta Otolaryngol 1993;113:62-7. https://doi.org/10.3109/00016489309135768.

[38] Stapczynski JS. Blast injuries. Ann Emerg Med 1982;11:687 - 94.

[39] Bass CR, Rafaels KA, Salzar RS. Pulmonary injury risk assessment for shortduration blasts. J Trauma 2008;65:604-15.

[40] Rafaels KA, Bass CRD, Panzer MB, Salzar RS. Pulmonary Injury Risk Assessment for Long-Duration Blasts : A Meta-Analysis. J Trauma 2010;69. https://doi.org/10.1097/TA.0b013e3181e88122.

[41] Panzer, M.B., Bass, C.R., Rafaels, K.A., Shridharani, J., Capehart BP. Primary blast survival and injury risk assessment for repeated blast exposures. $J$ Trauma Acute Care Surg 2012;72:454-66.

[42] Voort MM Van Der, Holm KB, Kummer PO, Teland JA, Doormaal JCAM Van, Dijkers HPA. A new standard for predicting lung injury in fl icted by Friedlander blast waves. J Loss Prev Process Ind 2016;40:396-405.

https://doi.org/10.1016/j.jp.2016.01.014.

[43] Rafaels KA, Bass CR, R. S. Salzar, Panzer MB, Woods WA, Feldman S, et al. Survival Risk Assessment for Primary Blast Exposures to the Head. J Neurotrauma 2011;28:2319-28. https://doi.org/https://doi.org/10.1089/neu.2009.1207.

[44] Rafaels KA, Bass CRD, Panzer MB, Salzar RS, Woods WA, Feldman SH, et al. Brain injury risk from primary blast. J Trauma Acute Care Surg 2012;73:895-901. https://doi.org/10.1097/TA.0b013e31825a760e.

[45] Trimble K, Clasper J. Anti-personnel mine injury; mechanism and medical management. J R Army Med Corps 2001;147:73-9. https://doi.org/10.1136/jramc-147-01-07.

[46] Dutch Safety Board. Mortar Accident Mali. The Hague: 2017.

[47] Jenzen-Jones NR. Submunitions in Syria: additions and updates 2013. https://rogueadventurer.com/2013/04/09/submunitions-in-syria-additions-andupdates/.

[48] FEMA. Risk Assessment - A How-To Guide to Mitigate Potential Terrorist Attacks Against Buildings. 2005.

[49] B Brismar LB. The Terrorist Bomb Explosion in Bologna, Italy, 1980: An Analysis of the Effects and Injuries Sustained. J Trauma Inj Infect Crit Care 1982;22:216-20. https://doi.org/10.1097/00005373-198203000-00007.

[50] Hill JF. Blast injury with particular reference to recent terrorist bombing 
J.W. Denny, A.S. Dickinson, G.S. Langdon. (2021). Defining blast loading 'zones of relevance' for primary blast injury research: A consensus of injury criteria for idealised explosive scenarios. (Preprint).

incidents. Ann R Coll Surg Engl 1979;61:4-11.

[51] Department of Defense. Report of the DOD Commission on Beirut International Airport Terrorist Act, October 23, 1983. 1983.

[52] Champion HR, Holcomb JB, Young LA. Injuries from explosions: Physics, biophysics, pathology, and required research focus. J Trauma - Inj Infect Crit Care 2009;66:1468-77. https://doi.org/10.1097/TA.0b013e3181a27e7f. 